Sociohistórica, nº 41, e047, 1er. Semestre de 2018. ISSN 1852-1606

Universidad Nacional de La Plata.

Facultad de Humanidades y Ciencias de la Educación.

Centro de Investigaciones Socio Históricas

\title{
Apuntes para una lectura de Carlos Matus desde los debates sobre "estilos de desarrollo"
}

\section{María Celeste Viedma *}

* Universidad de Buenos Aires - Centro Cultural de la Cooperación, Argentina

mcviedma@gmail.com

Cita recomendada: Viedma, M. C. (2018). Apuntes para una lectura de Carlos Matus desde los debates sobre “estilos de desarrollo". Sociohistorica, 41, e047. https://doi.org/10.24215/18521606e047 


\section{Apuntes para una lectura de Carlos Matus desde los debates sobre "estilos de desarrollo"}

Notes for a reading of Carlos Matus from the "development styles" debates

Maria Celeste Viedma

Universidad de Buenos Aires - Centro Cultural de la

Cooperación, Argentina

mcviedma@gmail.com

\section{Resumen:}

El artículo ofrece una lectura de la producción de Carlos Matus de fines de la década del sesenta y principios de los setenta a partir de su puesta en serie con los debates sobre estilos de desarrollo. Se busca tejer un diálogo en el que convergen la pregunta por la planificación del futuro para los países latinoamericanos, su factibilidad material y política y los estilos o estrategias de desarrollo. En primer lugar, se analiza el modo en que Matus concibió la planificación atendiendo tres dimensiones: estrategia, viabilidad política y desarrollo. Su puesta en relación con los debates sobre estilos de desarrollo se aborda en la segunda parte del artículo siguiendo las mismas dimensiones. El trabajo concluye en la necesidad de revisar los modos en los que se recupera la obra de Matus en la actualidad.

Palabras Clave: Carlos Matus, Planificación estratégica, Estilos de desarrollo.

\section{Abstract:}

The article offers a reading of the production of Carlos Matus from the late sixties and early seventies through its approach from the "styles of development" debates. It seeks to weave a dialogue in which the question for future planning in Latin American countries converges with their material and political feasibility and development styles or strategies. First, the article analyzes the way in which Matus conceived planning in three dimensions: strategy, political viability and development. Its relation with the "development styles" debates is addressed in the second part of the article following the same dimensions. The work concludes on the need to review the ways in which the work of Matus is recovered today.

KeYwords: Carlos Matus, Strategic planning, Development styles.

\section{INTRODUCCIÓN}

En los últimos años, distintos trabajos han recuperado las conceptualizaciones de Carlos Matus en torno a la noción de planificación estratégica para pensar las recientes transformaciones y potencialidades de los Estados latinoamericanos. Los trabajos aludidos procuran dar cuenta de la multiplicidad de actores que intervienen en el diseño y la gestión de las políticas (y, por ende, el carácter conflictivo que asumen estas) analizando, con mayor o menor énfasis, problemas como las capacidades de gobierno, la gobernabilidad y el proyecto de gobierno (Ossorio, 2003; Spinelli, 2012; Bernazza \& Longo, 2014; Neirotti, 2016). La recuperación de la producción de Matus se ve asociada, asimismo, a la puesta en agenda del problema del desarrollo de nuestros países y el rol que compete al Estado en los nuevos procesos ${ }^{1}$, dentro del cual la planificación adquiere particular relevancia (Sotelo Maciel, 2013). En virtud de esto último, en el presente trabajo procuraremos adentrarnos en algunos libros y documentos de Matus relativamente desatendidos hasta el momento, cuya recuperación consideramos fructífera por el modo en que en ella se articulan planificación, política y desarrollo. Efectivamente, las reflexiones actuales se han concentrado en términos generales en la producción del autor de los años ochenta y noventa, quedando relativamente descuidada la producción sobre la cual trabajaremos en este artículo, en la que la cuestión del desarrollo adquiere una centralidad que no puede apreciarse en sus trabajos posteriores. En virtud precisamente de la importancia 
que reviste el desarrollo en los trabajos tempranos de Matus, y el modo en que este se articula con la pregunta por la planificación del futuro y su viabilidad, es que resulta relevante su puesta en serie con los debates sobre estilos de desarrollo que le fueron contemporáneos. El aporte que nuestro trabajo se propone realizar es pues un primer acercamiento a dicho encuentro.

El trabajo está estructurado en dos grandes secciones: una primera parte dedicada al análisis de los escritos de Matus y, en segundo lugar, una visita a los debates sobre estilos de desarrollo con la que hacemos dialogar las reflexiones que la preceden. Trabajaremos en ambos casos con tres grandes nodos: estrategia o planificación en términos generales, la cuestión de la viabilidad política y el problema del desarrollo. Entendemos, como se verá a lo largo del artículo, que este último otorga sentido a los anteriores. Por último, presentamos algunas conclusiones en relación al modo en que actualmente se recupera la producción de Carlos Matus y esbozamos algunas líneas posibles para futuros abordajes.

\section{Primera parte: Planificación, política y desarrollo, Carlos Matus en los años SESENTA Y SETENTA}

El procedimiento estratégico arriba a una definición sobre la dirección del proceso de desarrollo y las formas limitadas de encauzar la realidad hacia ese rumbo. (...) De esa manera, por un lado, la estrategia como proyecto político es un elemento básico de la planificación, su pieza fundamental desde el momento en que allí se deciden las orientaciones principales, y por el otro, el procedimiento estratégico es una actitud y un método que obliga a una revisión coherente de las demás partes integrantes del sistema de planificación (Matus, 1972, p. 109).

A continuación presentamos la propuesta de planificación estratégica basándonos fundamentalmente en el primer libro de Carlos Matus: Estrategia y plan (1972). Publicado bajo la colección "Textos del Instituto Latinoamericano de Planificación Económica y Social” (ILPES), se trata de una de las últimas contribuciones de Matus como director de los Servicios de Asesoría de este Instituto ${ }^{2}$, antes de asumir como Ministro de Economía del gobierno de Salvador Allende en Chile. Ingresamos al problema por su puerta de entrada habitual, a saber: detallando las diferencias con respecto a la planificación normativa, para luego concentrarnos en los aspectos metodológicos, el problema de la viabilidad política y, por último, anudando lo anterior con el problema que resulta de nuestro especial interés: el desarrollo. Si bien seguiremos la línea argumental del libro aludido, procuraremos enriquecer el análisis con otros documentos elaborados en el seno del ILPES en los que Matus participó (ILPES, 1967, 1969 y 1970 y Matus, 1965).

\section{Planificación ESTRATÉgicA Y PLANIFICACión NORMATIVA}

La estrategia constituye "un análisis y un propósito de futuro" (Matus, 1972, p. 104) que supone la construcción de un modelo sobre el que se realizarán las simulaciones de las transformaciones deseadas. De este modo, un procedimiento será estratégico si procura definir un curso de acción a partir del ensayo de alteraciones sobre la realidad efectivamente existente de acuerdo al conocimiento que se tenga de ella. Es decir, las acciones no se definen a partir de la mera enunciación de las metas de transformación, sino en función de un análisis de su viabilidad. Por el contrario, un procedimiento normativo define las acciones necesarias en función de los objetivos definidos a priori, sin consideración de cuáles son las alteraciones que deben producirse en los procesos actuales para que esa transformación resulte viable. El autor diagnostica que la planificación se caracterizó hasta el momento por su carácter normativo, en vistas de su incapacidad para derivar cursos de acción sectoriales que pudieran dar cuenta de la complejidad de sus interrelaciones en una totalidad. Este supuesto epistemológico de la planificación normativa se complementa con el de la existencia de una discontinuidad histórica entre el pasado y el futuro planificado sobre el que se procura (y se cree posible) imprimir la máxima racionalidad. La planificación se asemeja, desde esta concepción, a la 
formulación de una norma (cómo debe ser la realidad), sin consideración del proceso por el cual se logrará llegar a ese resultado.

Ahora bien, en este trabajo quisiéramos concentrarnos en tres aspectos de la planificación estratégica. Por un lado, la necesidad de partir de la realidad existente para dar cuenta del proceso de transformación que se busca desencadenar obliga a transformar la metodología con que se planifica. Si la estrategia supone la definición de la dirección u orientaciones principales del proceso, el procedimiento estratégico implica revisar todas las partes integrantes y dar cuenta de sus relaciones en las distintas etapas de su trayectoria. Nos abocaremos a estas cuestiones en el siguiente apartado. En segundo lugar, el análisis de viabilidad supone consideraciones no sólo acerca de las transformaciones materiales, sino también sobre las posibilidades que la correlación de fuerzas sociales existente ofrece a quien planifica y cómo puede transformarse. Dedicaremos entonces particular atención a la noción de viabilidad política a la que Matus refiere. Por último, nos detendremos en el concepto que, a nuestro entender, anuda y otorga sentido a las reflexiones precedentes, a saber, la cuestión del desarrollo de los países latinoamericanos.

\section{EL PROCEDIMIENTO ESTRATÉGICO: DEFINICIÓN DE TRAYECTORIAS}

La estrategia supone no sólo la definición de una dirección o meta, sino también de una trayectoria definida en función de un diagnóstico inicial. La trayectoria implica formular metas para cada una de las etapas del proceso y sus aplicaciones concretas. Así, en primer lugar, la planificación estratégica implica tres niveles de abstracción: la exploración de las "grandes alternativas", la definición de las etapas o fases a partir de las cuales se alcanzarán las metas y, por último, las modalidades de ejecución concretas de cada etapa, partiendo de la situación inicial. Esto supone la integración de lo que, en un seminario dictado años antes de publicar el libro en cuestión, Matus denominaba mecanismos de orientación, de decisión y de ejecución, es decir, una instancia que tiene el propósito de funcionar como "guía", otra que transforma dichas proposiciones más generales "en una táctica para el presente" y por último una instancia de "detalle operativo" (Matus, 1965, p. 10). Una de las dificultades de la planificación normativa es precisamente su incapacidad para dar cuenta de esta operacionalización de los objetivos generales en una trayectoria.

La estrategia "obliga a pensar en términos de 'alteraciones concretas' y no abstractas, en proyectos sociales básicos y no simplemente en coeficientes o magnitudes económicas" (Matus, 1972, p. 111). No se trata únicamente de incorporar definiciones de corto plazo, entendidas como la desagregación del plan en períodos temporales más reducidos, sino de incorporar al análisis distintos niveles de abstracción. Los proyectos sociales básicos, elemento de máxima desagregación en la planificación, constituyen abstracciones de "primer grado" o "representaciones directas de una realidad singular (...) únicas por sus características y momento histórico" (Matus, 1972, p. 112). Son propósitos cuya singularidad deriva de su "correspondencia con un proyecto social" o su "concepción ideológico-económica" (Matus, 1972, p. 179). Por el contrario, las categorías de "segundo grado" constituyen generalizaciones universales, como por ejemplo, "las importaciones, exportaciones, inversiones, capital, ocupación, consumo, producto, etc.” (Matus, 1972, p. 112), y las de "tercer grado" refieren a los coeficientes que se construyen a partir de las segundas (tasa de crecimiento, etc.). Para Matus, la planificación no puede efectuarse únicamente a partir de las abstracciones de segundo y tercer grado, ya que entonces se privilegia la cantidad por sobre la calidad y queda implícita la definición de la dirección del proceso. Analizaremos más en profundidad estas consideraciones.

Uno de los supuestos epistemológicos de la planificación normativa es la existencia de correspondencia entre las categorías de análisis y la realidad, categorías que a su vez producen una separación "artificial" de las esferas económica, social y política, definiendo "una totalidad analítica de 'lo económico' amputada y, en consecuencia, autolimitante" (Matus, 1972, p. 77). La estrategia se caracteriza por integrar estas esferas y por distanciarse de la cuantificación extensiva a todos los procesos sociales, procurando la selección de variables estratégicas y considerando sus aspectos cualitativos. La crítica a las categorías económicas tradicionales se 
produce, por un lado, por su miopía para comprender la complejidad de la realidad social y, por el otro, por su carácter ideológico, perversamente disimulado bajo una supuesta neutralidad técnica. El procedimiento estratégico se opone al "modelo técnico-normativo, que define un concepto de plan donde la proyección responde a la eficacia técnica, subordinada siempre a las restricciones de alguna concepción ideológica implícita” (Matus, 1972, p. 104). Lejos de dificultar la labor técnica, la visibilidad de la ideología convierte la "racionalidad técnica" en "un instrumento más flexible puesto al servicio de la primera" (Matus, 1972, p. 105). Matus no desconoce la necesidad de cuantificar, pero advierte que la misma debe definirse en función de la elección explícita de una dirección. La definición de los proyectos sociales básicos, es decir, de los elementos operativos del plan en su máximo nivel de concreción, lejos de empobrecer la estrategia, funciona como garantía para no descuidar los aspectos cualitativos y mantener la elección sobre la dirección del proceso. Destacamos especialmente esta insistencia por visibilizar el posicionamiento ideológico en el proceso de planificación, ya que es un elemento que encontraremos presente también en los debates sobre estilos de desarrollo que abordaremos en la segunda parte del trabajo.

Ahora bien, los proyectos sociales básicos deben ser analizados en principio en su coherencia con la imagen-objetivo, metas últimas o definición de la dirección hacia la cual se quiere encauzar el proceso. Analizaremos algunas consideraciones en relación con la imagen-objetivo en el apartado siguiente. En un segundo momento, deben desagregarse ahora sí las etapas y su ordenamiento temporal y volver a analizarse, a su vez, la coherencia de los proyectos para cada una de las etapas entre sí. La desagregación del proceso en etapas exige la adopción de una perspectiva dinámica en la que los proyectos y las metas deben estudiarse y revisarse en cada momento. El diagnóstico y el análisis de viabilidad no pueden realizarse de una vez y para siempre al comienzo, sino que son parte integrante de todas las etapas. Así, la planificación estratégica no opone corto y largo plazo, ni planificación total o sectorial, sino que procura su integración en un proceso dinámico. Por último, este dinamismo garantiza que podrán incorporarse los elementos desconocidos e incluso impensables al comienzo en las etapas posteriores, dando lugar a la consideración de lo imprevisible en la planificación. El análisis implica, de este modo, no sólo la viabilidad de los proyectos sino de una secuencia específica.

Señalemos finalmente que, en un nivel procedimental, las consideraciones anteriores se aplican a una simulación sobre la realidad. Es decir, a partir del diagnóstico, se realizan las proyecciones en etapas sucesivas hasta alcanzar la imagen deseada. Como vemos, esto significa que el procedimiento estratégico requiere considerar una gran cantidad de variables y sus relaciones. Es decir que su realización representa un desafío en términos de la cantidad de información que se debe relevar para poder realizar el diagnóstico y la simulación, cuestión señalada por el mismo Matus en trabajos anteriores (ILPES, 1969 y Matus, 1965). Con respecto a esta dificultad, el autor indica que el lenguaje matemático podría enriquecer el análisis y se detiene en los avances realizados en la materia por Oscar Varsavsky, cuestión sobre la que volveremos en la segunda parte de este artículo ${ }^{3}$. Nos limitaremos a señalar por el momento que, para Matus, dada la necesidad de considerar elementos cualitativos y el estado incipiente de la formalización matemática en lo que respecta a las ciencias sociales, hasta tanto no se realicen mayores avances en este campo será conveniente continuar utilizando el lenguaje verbal y evitar la excesiva cuantificación.

Hasta aquí, hemos descrito brevemente en qué consiste el procedimiento de análisis de viabilidad, pero nos reservamos la consideración acerca de a qué refiere puntualmente esta última, cuestión que desagregaremos en el próximo apartado.

\section{Planificación y grupos SOCIALES: EL PROBLEMA DE LA VIABILIDAD POLÍTICA}

Anteriormente señalamos que una de las características de la planificación estratégica es la integración de los aspectos económicos, sociales y políticos. Esto se relaciona con otro aspecto central del análisis, a saber: la planificación no puede concebirse como un procedimiento tendiente a eliminar "desajustes" o 
"desequilibrios", puesto que estos son constitutivos de la realidad social en la medida en que expresan intereses de distintos grupos sociales en pugna. La consideración de la existencia de grupos sociales en conflicto es un elemento estructurante de la argumentación de Matus: la pregunta por la viabilidad supone la consideración de la existencia de sectores sociales y políticos que se opondrán a la propia estrategia y con los cuales será necesario confrontar o negociar, al tiempo que implica la necesidad de construir consenso con otros grupos aliados para alcanzarla. Estas consideraciones deben incorporarse a todo proceso de planificación que procure ser exitoso.

Las transformaciones tendrán así dos planos complementarios sobre los cuales debe analizarse la viabilidad: por un lado, la acción material referida a la creación de las condiciones concretas para el cambio y, por el otro, el proceso intelectual de formación de consenso, si se trata de una estrategia oficial, o bien de conciencia, si se trata de una estrategia de oposición. Ambos aspectos definen consideraciones sobre la viabilidad material en el primer caso y viabilidad política en el segundo. El dinamismo que debe imprimirse al procedimiento estratégico garantiza que aquello que puede resultar inviable en un determinado momento inicial, pueda convertirse en viable material o políticamente a partir de las aproximaciones sucesivas en las distintas etapas del proceso. Aunque esto no vuelve necesarios los cambios, ya que la viabilidad depende de las condiciones históricamente construidas de las que se parte. Matus parafrasea en este punto a Karl Marx y señala que "la viabilidad no es, sino que se construye; pero su construcción depende de las condiciones de cada momento histórico" (Matus, 1972, p. 121). En virtud del carácter histórico de la viabilidad, el aspecto político adquiere mayor relevancia en la medida en que la viabilidad material también se relaciona con la existencia de fuerzas sociales en conflicto. Sin embargo, como veremos, la posición material de los grupos sociales no determina a priori su posicionamiento político, sino que este último depende del grado de información y de toma de conciencia de los grupos.

El análisis de viabilidad política supone la incorporación de los grupos sociales a los procedimientos mencionados en el apartado anterior, definiéndose en primer lugar para cada grupo un grado de cohesión, crecimiento y avance en cada etapa del proceso. El primero refiere a la capacidad de aglutinar a los grupos sociales que puedan apoyar la propia estrategia, que dependerá a su vez de las capacidades para influir sobre la toma de conciencia en relación al proceso de ejecución del plan y sus metas. El crecimiento supone agregar nuevas fuerzas sociales a la misma, está supeditado a los éxitos obtenidos y también conlleva un esfuerzo por poner la información correcta a disposición de los distintos grupos y su toma de conciencia en relación a esta. El avance, por su parte, refiere a la búsqueda de nuevas metas sobre los apoyos obtenidos y supone la obtención de una ventaja hacia la consecución de las metas últimas.

En segundo lugar, debe definirse el grado de influencia o poder de cada grupo, es decir, su capacidad para imponerse y su grado de compromiso, que refiere a qué tan establecido se encuentra su apoyo u oposición al plan o su flexibilidad con respecto a cada uno de los proyectos. La influencia puede referirse a: acceso de los niveles de decisión, capacidad para formar opinión y control para resistir, obstruir o vetar proyectos. El análisis exige también entonces la consideración de cuáles serán los grupos que desarrollarán una contraestrategia, es decir, que buscarán impedir la realización de cada uno de los proyectos por separado y de las metas últimas en términos generales.

Cabe recordar, en relación al apartado anterior, que la estrategia supone la identificación de cuáles serán los grupos que podrán respaldar las metas últimas en términos generales para todo el proceso, y a su vez para cada una de las etapas de la trayectoria y en relación a cada uno de los proyectos básicos. Un mismo grupo puede posicionarse favorablemente ante ciertos proyectos y no otros, o en ciertos momentos y no en otros. A partir de estas consideraciones, se traza una trayectoria que dependerá entonces de la posición que asuma cada uno de los grupos frente a cada proyecto y en cada una de las etapas. Por otra parte, los avances en dirección a las metas últimas no pueden evaluarse en forma lineal, puesto que existen estrategias opuestas en conflicto; toda trayectoria será necesariamente fluctuante, registrando momentos de avance y momentos de retroceso. 
Ante la pregunta por cómo se definen y caracterizan cada uno de los grupos, Matus señala que su clasificación puede desprenderse directamente de la estrategia definida realizando la pregunta, para cada uno de los proyectos básicos, por "quiénes resultarían beneficiados y quiénes perjudicados" (Matus, 1972, p. 162). Es decir, el análisis de viabilidad política no exige asumir una única definición de estratificación social, sino que basta con que se identifiquen al comienzo los grupos que se supone podrán oponerse o mostrarse favorables a cada proyecto y luego verificar en el análisis de la trayectoria la utilidad de estas definiciones. Sin embargo, el autor señala que en términos generales deberá tomarse en consideración la estructura institucional sobre la que se procura aplicar la estrategia, los grupos sociales relativamente estructurados (sindicatos, partidos políticos) y la conducta colectiva de las partes de la sociedad que no se encuentran organizadas. El primer aspecto, referente a la estructura institucional sobre la que la planificación se inserta, a saber, la estructura administrativa pública, merece especial atención. Para Matus, pueden distinguirse dos grupos fundamentales: las estructuras políticas y la burocracia, que a su vez incluye sectores técnicos establecidos en el sistema. Quienes procuren promover la planificación deberán también generar consenso en ambos grupos en torno a la necesidad de la misma. Estos sectores poseen distintos criterios de eficacia que, en ausencia de una concepción que integre el análisis económico, político y administrativo, tenderán a entrar en conflicto. Así, el criterio de eficacia económica (alternativa más económica para alcanzar un objetivo) no puede quedar disociado de la eficacia política (afianzar y acrecentar el poder) ni tampoco del criterio de eficacia administrativa (cumplimiento de la norma en los procedimientos). Una de las principales dificultades de la planificación hasta entonces fue, de acuerdo con el autor, la disociación y conflicto entre los grupos mencionados.

Por último, señalemos que uno de los elementos que contribuyen a la viabilidad política de la planificación es la participación social. Matus hacía referencia al mismo requisito en un seminario previo, señalando que "la planificación no puede ser un acto técnico aislado de la sociedad" (Matus, 1965, p. 9). Para que la generación de consenso entre los grupos sociales afines al proyecto sea posible, no basta con que se tomen en consideración sus intereses en la definición de la trayectoria, sino que la misma construcción de ésta última debe hacerse convocándolos. Para lograr una participación efectiva, es decir, que incluya a los vastos grupos sociales y no sólo a los "más poderosos o mejor representados ante al gobierno" (Matus, 1972, p. 165), debe procurarse una cierta descentralización en el proceso, garantizar la difusión de información sobre el plan, intervenir para organizar a los grupos sociales que se encuentren dispersos y buscar su pronunciamiento en relación a los objetivos del plan. Incluso en aquellos casos en los que el apoyo se encuentre "motivado por costumbres, prejuicios, factores emotivos, etc." (Matus, 1972, p. 167), debe promoverse una discusión conjunta para lograr un pronunciamiento racional. En Estrategia y plan (1972), Matus no ofrece mayores precisiones acerca de cómo podría instrumentarse la participación social en cada una de las etapas del proceso de planificación. Sin embargo, en el seminario mencionado anteriormente (Matus, 1965) sugiere la convocatoria de las organizaciones sociales fundamentales para la etapa de definición de las metas últimas y una participación comunal y más directa para las definiciones intermedias: "en las definiciones anuales y en el proceso de ejecución de los planes, la participación popular puede y debe ser intensa y masiva para llenar un vacío que las formas tradicionales de expresión popular partidaria no cubren” (Matus, 1965, p. 14).

Hasta aquí, deliberadamente nos hemos referido a la planificación en términos generales. Quienes se encuentren familiarizados con los trabajos posteriores del autor seguramente hayan encontrado vastas similitudes. Sin embargo, estas tempranas reflexiones en torno a la planificación estratégica adquieren sentido en el marco de una pregunta más general por el desarrollo de los países latinoamericanos, como veremos en el próximo apartado. 


\section{Planificación y estrategias de DesarRollo}

En las secciones anteriores, hicimos referencia a la necesidad de definir la imagen-objetivo o metas últimas hacia las que procura dirigirse al proceso de planificación. Señalamos también que la definición de estas "metas últimas" supone un posicionamiento ideológico que debe hacerse explícito definiendo cualitativamente los objetivos perseguidos. Para Matus, como señalamos, las metas de la planificación no pueden ser abstracciones tales como "alcanzar determinada tasa de crecimiento", al tiempo que los aspectos económicos deben integrarse con los políticos y sociales. Estas consideraciones se derivan en efecto de la crítica del autor, señalada en la primera parte del libro, a la consideración de la velocidad del crecimiento económico como único objetivo de la planificación tradicional y medida del desarrollo de los países. Veamos.

En la primera sección de Estrategia y plan (1972), Matus señala que la formación de oficinas de planificación en la mayor parte de los estados latinoamericanos iniciada luego de la segunda posguerra estuvo relacionada con el consenso político en relación a la necesidad de conducir y abordar las dificultades que presentaba el desarrollo por sustitución de importaciones. En virtud de ello, los grupos intelectuales y técnicos se abocaron al problema de la velocidad del crecimiento, quedando implícito el consenso en torno a la dirección del proceso de desarrollo:

El concepto de dirección del proceso de desarrollo implica la definición de un proyecto social, que a su vez supone una estructura de relaciones de poder, un sistema básico de decisiones, un patrón de relaciones con el exterior y una definición precisa sobre las relaciones sociales de producción que caracterizan la sociedad que se busca construir o se pretende alcanzar (Matus, 1972, p. 15).

Ahora bien, Matus señala que, hacia fines de la década del sesenta, resulta evidente que los beneficios augurados por la sustitución de importaciones y la planificación que se apoyó sobre ella no fueron extensivos a todos los grupos sociales. Vastas regiones quedaron postergadas y grandes mayorías marginadas del proceso de modernización. Es así que comenzó a circular entre distintos grupos sociales la preocupación en torno a qué dirección debe tomar el proceso de desarrollo.

Las críticas del autor a la planificación normativa desarrolladas en los apartados anteriores se comprenden cabalmente a la luz de estas precisiones. Por un lado, no es de extrañar que se utilizaran categorías económicas, sin considerar los procesos sociopolíticos, si la preocupación que gravitaba sobre los análisis y proyecciones realizados era precisamente la velocidad del crecimiento económico. La incorporación a los estudios posteriores de conceptos provenientes de otras disciplinas como la sociología o la ciencia política se relaciona con la pérdida de consenso en torno a la dirección del desarrollo. Surgen entonces nuevas categorías que se aproximan a pensar el problema de la dirección: “'sector moderno' y 'sector no moderno', 'estrategia', 'modelo consumista', 'patrón o estilo de desarrollo', 'crecimiento por diversificación', 'crecimiento por ampliación', 'saturación rural', 'desarrollo horizontal y vertical', 'marginalidad', 'grado de dependencia', 'imagen-objetivo', etc." (Matus, 1972, p. 21). Nótese la mención a la noción de estilos de desarrollo, sobre la que volveremos en la segunda parte del artículo. Tampoco resulta extraño que las consideraciones acerca del problema del gobierno y el consenso, es decir, la preocupación por la viabilidad política de los planes, quedara fuera del campo de la planificación en el marco de un contexto de acuerdo en los distintos grupos sociales en torno al proceso de desarrollo vigente: "de ahí que durante las primeras etapas predomine un criterio de proyecciones a mediano o a largo plazo de 'lo económico', más que un concepto amplio de acción de gobierno” (Matus, 1972, p. 20).

Por otra parte, como vimos anteriormente, la planificación requiere de la definición de objetivos y metas últimas. En otras palabras, "no puede operar sin una política de desarrollo definida y aceptada" (Matus, 1972, p. 22). Esto significa que, para Matus, los problemas de la planificación son en su mayoría producto de la falta de consenso en torno a la definición de un nuevo patrón de desarrollo. El mismo posicionamiento se evidencia en una reunión del ILPES de la que Matus participó en 1968: en su intervención, el economista señala la necesidad de definir "estrategias nacionales de desarrollo" para mejorar la operatividad de la 
planificación e "ir concretando la imagen del desarrollo posible y de los grandes cambios de política económica necesarios" (ILPES, 1968, p. 8) ${ }^{4}$. Esta falta de definición conlleva la "pérdida de contenido sustantivo de los planes" (Matus, 1972, p. 23) y el aislamiento de las oficinas de planificación con respecto a los centros de decisión política. En palabras de nuestro autor:

La llamada crisis de la planificación, en suma, no es más que un subproducto de la crisis del desarrollo latinoamericano. Esta última es en gran parte la consecuencia de condiciones materiales, pero también está determinada por la crisis del pensamiento que no puede alimentar fuerzas que contrarresten esas condiciones materiales (Matus, 1972, p. 24).

Con estas afirmaciones, Matus se distancia de las observaciones de algunos de sus contemporáneos acerca de que las dificultades de la planificación provienen de la calidad de sus procedimientos formales, a saber: la falta de planes anuales operativos, las rigideces de la administración pública, la insuficiencia de sistemas de información e incluso la falta de ofertas de financiamiento externo o la incertidumbre del comercio exterior. Debemos señalar, no obstante, que el autor se había hecho eco de estas discusiones algunos años antes en los que presentaba varias de estas dificultades como parte de los problemas fundamentales de la planificación (Matus, 1965). En 1972, sin embargo, Matus consideraba que, si bien puede que dichos factores sean más visibles, eran en realidad efectos de la situación descrita más arriba. ${ }^{5}$

Ahora bien, ¿qué rol le cabe a la planificación en el escenario presentado? Matus rápidamente desecha una posible separación entre la esfera de la decisión política y la implementación técnica. La planificación no puede quedar reducida a la esfera técnica, pues de ese modo se "haría depender el plan, cuya nota fundamental es la modificación consciente de un proceso, de las condiciones políticas propicias para el cambio, que emergerían del curso natural del proceso" (Matus, 1972, p. 25). En este sentido, la propuesta de la planificación estratégica, al constituir una síntesis entre el análisis económico y el político, se ofrece para el autor como una herramienta valiosa para impulsar una política de desarrollo. Esto en tanto permite resolver el problema de la distinción entre las condiciones históricas bajo las cuales se desenvuelve la planificación (denominada coyuntura dinámica) del conjunto de ideas que se quieren llevar adelante (política construida) y ofrece una modalidad específica para analizar qué posibilidades tiene esta última de convertirse en la primera, es decir, su viabilidad.

Por último, señalemos que la afirmación de que en la planificación deben considerarse integralmente los aspectos económicos, sociales y políticos es tributaria de una concepción del desarrollo que debe exceder los aspectos meramente económicos y "situar el desarrollo dentro de una perspectiva de la mayor universalidad posible” (Matus, 1972, p. 80). Por otra parte, recordemos que, como señalamos anteriormente, si la estrategia implica que la selección de proyectos sociales básicos debe basarse en la imagen-objetivo, con la cual estos deben guardar coherencia, dicha imagen refiere precisamente a la dirección del proceso de desarrollo. Esto significa que el patrón de desarrollo perseguido no puede reducirse a un puñado de indicadores, como ocurría con la planificación de las décadas precedentes:

en muchas oportunidades esas cuantificaciones suelen hacerse como algo previo a la definición cualitativa de un patrón o estilo de desarrollo. En varios casos, además, la definición de alternativas en los planes tiende a transformarse en el análisis de variantes numéricas (ritmos de crecimiento alternativos), y no en el de los beneficios y costos de patrones de desarrollo cualitativamente diferentes $^{6}$ (Matus, 1972, p. 112).

Por el contrario, la imagen-objetivo constituye, precisamente, "el 'faro' del proceso de desarrollo, es la señal que recuerda y anuncia el peligro de extraviar la dirección” (Matus, 1972, p. 169). La denominación no es caprichosa, puesto que es un "punto de referencia relativo en el futuro" (Matus, 1972, p. 171), la imagen-objetivo no debe ser necesariamente exhaustiva o extenderse a todos los aspectos de la vida social ni tampoco establecer plazos rígidos para su cumplimiento, aunque sí "debe aspirar a construir un proyecto social integrado, nacional o regional” (Matus, 1972, p. 173). Tampoco puede la imagen-objetivo elaborarse en base a una "visión tecnocrática sin arraigo en ningún grupo social significativo", sino que "cabe preguntarse quién formula y a qué responde" (Matus, 1972, p. 174). La representatividad es pues un requisito que debe 
cumplir la imagen-objetivo y constituye su única forma de evaluación, no siendo susceptible de ser objetada técnicamente. Su consistencia interna, es decir, la coherencia entre sus proposiciones, puede ser objeto de crítica, pero no su relación con los problemas sociales o su validez en sí mismas, puesto que se trata de una definición ideológica.

Para finalizar con esta primera parte, antes de abordar los debates sobre estilos de desarrollo, quisiéramos detenernos brevemente en las discusiones en las que el mismo Matus participó en torno a cuál debe ser la estrategia de desarrollo para los países latinoamericanos.

\section{Hacia una estrategia de desarrollo interior para América latina}

Antes de la puesta en circulación de Estrategia y plan, Matus participó en una publicación del ILPES titulada Dos polémicas sobre el desarrollo de América Latina (1970) con cuatro contribuciones. Allí, aborda el problema del desarrollo interior de los países latinoamericanos, en referencia a la localización geográfica de la actividad económica, correspondiente a su vez con un modelo de desarrollo "hacia adentro". Se trata, según el autor, de "un interrogante fundamental para esclarecer una estrategia de desarrollo para la región latinoamericana” (Matus, 1970a, p. 3). Nos interesa rescatar algunas de sus concepciones acerca del desarrollo para ponerlas en relación con los debates que recuperaremos en la segunda parte de este artículo.

Para Matus, un modelo de desarrollo "vertical", basado en la prosperidad económica de las zonas costeras por estar vinculadas con los países desarrollados hacia los cuales se orienta la actividad, no permite aprovechar adecuadamente los recursos naturales al tiempo que impide a los países latinoamericanos "fijar su propio patrón y ritmo de desarrollo de acuerdo a las conveniencias de la región" (Matus, 1970a, p. 9). Para el autor, la capacidad para decidir acerca del patrón de desarrollo que se considere conveniente y la soberanía sobre los propios recursos naturales son aspectos insoslayables para los países latinoamericanos. El patrón de desarrollo interior y horizontal, en la medida en que permite el desarrollo de las regiones menos desarrolladas de los países y se centra en la demanda interna, es compatible también con la integración regional latinoamericana. La estrategia de desarrollo vigente (a saber: vertical, orientada "hacia afuera") resulta ilógica e inviable en la medida en que condena a "la marginalización de las grandes masas" (Matus, 1970b, p. 28). Esto último constituye no sólo un problema social sino también una "subutilización de la capacidad humana de creación" (Matus, 1970b, p. 52). Veremos en la segunda parte del trabajo que el problema de la creatividad resulta fundamental para distinguir estilos de desarrollo alternativos.

Por otra parte, el modelo orientado hacia afuera genera también una "sustitución no consciente ni crítica de los valores propios por valores, adecuados o inadecuados, de una sociedad industrial avanzada" (Matus, 1970c, p. 94). Los centros desarrollados generan así una "atracción” sobre los países latinoamericanos que los condena a la dependencia cultural y tecnológica. Matus propone, por el contrario, la adopción de una estrategia centrada en los valores y pautas de consumo propios. Tanto la política tecnológica como las formas de consumo deben decidirse a partir de la definición de una estrategia de desarrollo. El problema de las pautas de consumo merece para Matus especial atención: estas deben definirse en función de las mayorías y no de las elites. Por lo tanto, sugiere una reducción de la oferta en pos de la masificación de ciertos bienes y servicios, accesibles para toda la población. El mercado se amplía pero no se diversifica en la medida en que una canasta más reducida contribuye a satisfacer las necesidades de las mayorías.

Veremos a continuación que algunas de estas consideraciones acerca del "patrón o estilo de desarrollo" y las modalidades que tendría que asumir su planificación y estudio de viabilidad guardan estrecha relación con debates contemporáneos a esta temprana producción de Carlos Matus. 


\section{SEGUNDA PARTE: LOS DEBATES SOBRE ESTILOS DE DESARROLLO}

Ya entre los mismos planificadores son muchos los que se preguntan “¿desarrollo para qué?”, “¿qué va a contener ese PBI?”, “¿qué le va a tocar a cada uno?”. Conformarse con proponer una alta tasa de crecimiento es en esencia pedir "más de lo mismo" socialmente, lo cual puede ser suficiente definición para quienes estén satisfechos con este sistema social, pero no para quienes quieran cambiarlo (Varsavsky, 1971a, p. 25).

Hacia fines de los años sesenta, poco tiempo antes de la publicación de los libros analizados, tuvo lugar tanto en América Latina como en buena parte del mundo desarrollado un debate álgido en torno a la "primera década del desarrollo". En 1968 desde Naciones Unidas se formuló una agenda de iniciativas para el Segundo decenio del desarrollo que destacó por la preocupación por la distribución del ingreso y el problema ambiental. En este marco, desde América Latina surgieron posiciones que se centraron en la postulación de los límites socioeconómicos del estilo de desarrollo existente. Estas formulaciones se erigieron en directa oposición a los "límites naturales al crecimiento" augurados por el Club de Roma, cuyos resultados fueron publicados por Dennis Meadows en 1970. Frente a los diagnósticos sombríos que desde el primer mundo pregonaban por un freno al crecimiento de los países desarrollados y un control de la natalidad para el mundo subdesarrollado, desde diversas instancias latinoamericanas se propusieron estilos de desarrollo alternativos y se elaboraron modelos en un esfuerzo por demostrar su factibilidad. Concretamente, en este trabajo nos centraremos en los primeros trabajos producidos desde la CEPAL, en el Centro de Estudios del Desarrollo (CENDES) venezolano y en el Instituto del Cálculo de la Universidad de Buenos Aires bajo la dirección de Oscar Varsavsky y, por último, en las contribuciones lideradas por Alfredo Eric Calcagno y sistematizadas luego de sus clases en la Facultad Latinoamericana de Ciencias Sociales (FLACSO).

Destacaremos tres aspectos de estas discusiones que entendemos resuenan en los desarrollos de Carlos Matus acerca de la planificación. En primer lugar, las críticas al desarrollo entendido en términos singulares y en sus aspectos exclusivamente económicos. En segundo lugar, nos abocaremos a la construcción de modelos propuesta por estas iniciativas para demostrar la factibilidad de los estilos de desarrollo alternativos. Por último, nos centraremos en un tipo de modelo en particular propuesto por Alfredo Eric Calcagno, Pedro Sainz y Juan de Barbieri para analizar en particular el problema de la viabilidad política.

\section{El “Otro DESARrollo”}

Como mencionamos, en oposición a los diagnósticos de Meadows que naturalizaban los límites al crecimiento, los discursos sobre el "otro desarrollo" realizan una operación de politización al postular que los límites al desarrollo son sociales y políticos. Esto tiene como resultado la aparición recurrente de frases en plural ("estilos", "modelos", "pautas", etc.) que desestabilizan todo efecto de unidad autoevidente de "el desarrollo", en singular, pensado generalmente como "mero crecimiento económico" y asociado a un "estilo de vida consumista”. Desarrollaremos más en profundidad estas afirmaciones.

En efecto, "estilos de desarrollo" significa que "hay muchos futuros posibles" (Varsavsky, 1971a, p. 9), aunque el estilo imperante se caracterice por tener por único objetivo el aumento de la tasa de crecimiento. La invitación a pensar otros estilos conlleva la impugnación a las categorías de la ciencia económica como única medida del desarrollo y una invitación a pensar los objetivos o metas del desarrollo en términos de otro tipo de variables. Estas consideraciones son particularmente fuertes en la propuesta de Proyectos Nacionales (1971) de Oscar Varsavsky:

Un Proyecto Nacional no debe plantearse en términos de tasas de crecimiento u otros indicadores cuantitativos globales, sino en términos de cumplir -a lo largo de un período apreciable de tiempo- un conjunto de diversos objetivos simultáneos que expresen con claridad todos los aspectos ideológicos. Para ello estos objetivos deben definirse, primero, en sus características cualitativas y sólo después cuantificarse para los distintos grupos de población (Varsavsky, 1971a, p. 25). 
Como puede apreciarse, encontramos aquí varios puntos de confluencia con el planteo de Matus analizado en la primera parte del artículo. Se impugnan las medidas económicas por su presunta neutralidad técnica, politizándolas y proponiendo la formulación de objetivos de carácter cualitativo cuyo posicionamiento ideológico resulte explícito. Sin embargo, notamos que Varsavsky refiere a Proyectos Nacionales, mientras que en Matus se destaca la insistencia por la búsqueda de una estrategia regional de desarrollo. El científico presenta algunas reservas en cuanto a la posibilidad de definir proyectos regionales, aunque en ambos casos la relación centro-periferia aparece como estructurante de la definición de estilos o estrategias de desarrollo ${ }^{7}$.En cuanto a la definición de los objetivos, Varsavsky propone la consideración de todas las necesidades humanas -materiales, políticas, sociales, espirituales, etc.-; Matus, por su parte, refiere a los aludidos proyectos sociales básicos, cuyo carácter no refiere precisamente a necesidades humanas consideradas en términos tan amplios como para Varsavsky, pero que frente a las "abstracciones de segundo grado" o "indicadores cuantitativos globales" definen propósitos históricamente situados cuya selección es producto de un posicionamiento ideológico. Como veremos en el siguiente apartado, la propuesta de cuantificar los objetivos para calcular su viabilidad no puede concebirse sin ser remitida a "metas últimas", sean las necesidades que sostienen el Proyecto Nacional, sea la imagen-objetivo a la que remite la estrategia de desarrollo elegida en el caso de Matus.

Otro aspecto a destacar con respecto al "otro desarrollo" es su impugnación al "estilo consumista" vigente que equipara "ciudadano" a "consumidor" y alienta formas de consumo suntuario como signo de prestigio. El carácter foráneo e impuesto de las "pautas de consumo" ${ }^{8}$ correspondientes a los países desarrollados se caracteriza por presentar una oferta altamente diversificada pero accesible para las elites y se opone, en efecto, a una estrategia de desarrollo que privilegie el acceso de las grandes mayorías a consumos relacionados con la satisfacción de necesidades (Varsavsky, 1971a). Planteos similares se presentaron en el apartado anterior, referido a las consideraciones de Matus acerca del problema del consumo.

Otro de los problemas recurrentes en los debates que analizamos es la adopción de estilos tecnológicos, o bien, estrategias de desarrollo de tecnologías que guarden relación con el estilo de desarrollo deseable y que no constituya, al igual que en el caso de las pautas de consumo, un producto de seguir pautas ajenas (Varsavsky, 1971a, p. 112). En efecto, el "estilo consumista" y el "estilo seguidista" se superponen, en virtud de que el primero es el ofrecido por los países desarrollados como modelo a seguir (Varsavsky, 1971b). Como vimos en el apartado anterior, también para Matus la adopción acrítica de valores foráneos constituía un problema que era necesario abordar para lograr una estrategia de desarrollo propia.

Por último, otro de los aspectos en los que las propuestas de Matus se articulan con los debates sobre estilos de desarrollo es en la insistencia por "pensar el futuro", en particular por dar cuenta de la factibilidad de "futuros más deseables; menos probables, tal vez, pero posibles" (Varsavsky, 1971a, p. 9). Es decir, se trata de la oposición a posicionamientos que se basen, como Meadows, en la fatalidad naturalizada del curso de la historia. Al decir de Matus: "mientras no se demuestre que alguna ley todavía no enunciada rige la evolución de la sociedad hacia un fin y una estructura determinados, toda imagen-objetivo será en cierta medida una utopía y toda estrategia, una posibilidad incierta” (Matus, 1972, p. 192).

\section{Modelos MATEMÁticos Y ESTUdios DE VIABILIDAD}

Los debates que analizamos se caracterizaron por proponer estilos de desarrollo alternativos a partir de la demostración de su viabilidad utilizando la experimentación numérica. Oscar Varsavsky, retomando la tesis de Edward Holland que aplicaba un modelo de experimentación numérica a la India, formalizó por primera vez, junto a Carlos Domingo en el Instituto del Cálculo, un modelo de Utopía de Tomás Moro en el año 1963, dando inicio a una serie de trabajos que utilizarían este método. En rigor, se trataba de un dispositivo que, haciendo uso de la naciente cibernética, permitía contemplar una gran cantidad de variables en forma simultánea para proyectar escenarios y calcular la viabilidad de determinadas líneas de acción. Buena 
parte de los trabajos realizados entonces pueden encontrarse en la compilación hecha por Oscar Varsavsky y Alfredo Eric Calcagno titulada América Latina: Modelos matemáticos. Ensayos de aplicación de modelos de experimentación numérica a la política económica (1971). El modelo permitía calcular no sólo la viabilidad material de un estilo sino también su viabilidad política. Desarrollaremos en primer lugar sus características en términos generales y dedicaremos el último apartado a la cuestión política.

Ya señalamos en la sección anterior las reservas que tenía Carlos Matus respecto de los modelos de experimentación numérica. Este autor señalaba que "la cibernética puede ser de gran utilidad en la formulación y conducción de estrategias de desarrollo, pero parece resultar insuficiente en su estado actual de desarrollo" (Matus, 1972, p. 149, el subrayado es nuestro). Matus también advertía la vacancia para el desarrollo de una nueva matemática que pudiera resultar adecuada para las ciencias sociales y sugería a matemáticos, politólogos, sociólogos y economistas darse a la tarea para su elaboración (Matus, 1972). El autor citaba en su libro al mismo Oscar Varsavsky, quien también destacaba que la matemática ortodoxa, construida para las ciencias naturales, no podía dar respuesta a los interrogantes de las ciencias sociales, pero esto en modo alguno significaba que no pudieran desarrollarse nuevos modelos más apropiados.

Independientemente de su posicionamiento en torno a la experimentación numérica, nos interesa señalar algunos puntos de cruce que encontramos significativos entre las propuestas de Matus y las presentes en estos debates. En primer lugar, la insistencia por encontrar un modo de calcular la viabilidad de los estilos o estrategias de desarrollo a través de un ejercicio de simulación que permitiera ensayar cursos de acción diversos sobre un modelo que, se supone, reacciona de manera análoga a la realidad social. Como vimos, el procedimiento estratégico supone, en efecto, dicho ejercicio, aunque de un modo menos formalizado que en los debates sobre estilos de desarrollo. En segundo lugar, es un requisito indispensable de ambos procedimientos, como señalamos previamente, que toda cuantificación remita a los objetivos o metas últimas, sea que se trate de las "necesidades", el "Proyecto Nacional" o bien la "imagen-objetivo". Es por eso que "los aspectos cualitativos del desarrollo pasan a primer plano y lo curioso es que comience a hacerse con métodos numéricos" (CENDES, 1971, p. 118). Para garantizar esta coherencia, cada una de las metas debe definirse de manera concreta: formularse como "abstracción de primer grado" para Matus, o bien, para Varsavsky "ser tan explícita y concreta" (Varsavsky, 1971a, p. 37) que permita conocer los recursos que requiere, el grado en que satisface las expectativas y los efectos que pueda tener sobre el resto de las metas. El análisis de viabilidad involucra, en síntesis, un análisis de coherencia en relación a objetivos últimos que sólo pueden objetarse en virtud de su carácter ideológico específico y no en función de criterios técnicos o neutrales: "al dar mayores prioridades a ciertas necesidades que a otras se las estaba evaluando éticamente, y esa evaluación es la que constituye la 'ideología' del 'estilo"' (Varsavsky, 1971b, p. 1015). Por último, otra característica en común es que, en función de lo dicho anteriormente, se trata de propuestas que buscan correrse de las discusiones sobre si debe prevalecer el largo o el corto plazo. Antes bien, se trata de proponer objetivos de largo plazo que sirvan como criterios para evaluar las políticas a corto plazo (Varsavsky, 1971b).

\section{Estilos DE DESARRollo, PROGRAMA DE GOBIERNO Y VIABILIDAD}

Quisiéramos señalar, por último, algunas consideraciones acerca de la viabilidad política de los estilos de desarrollo. Al respecto, Varsavsky señala que cada uno de los grupos sociales tiene expectativas, cierto nivel de satisfacción, alguna visibilidad de sus necesidades, cierta capacidad de espera para satisfacerlas, una determinada propensión a actuar, un cierto grado de organización (estructura institucional), composición ideológica, fuerza, conciencia (Varsavsky, 1971a). A su vez, son diversas las instituciones con algún grado de control del poder político y pueden transformarse de diferentes modos. Para este autor, el análisis en profundidad de todas estas consideraciones "llevaría tanto tiempo que significaría renunciar a la acción", mientras que no hacer ningún análisis sería "irracional” (Varsavsky, 1971a, p. 54). Sugiere, por lo tanto, un método que permita una aproximación al problema. En efecto, en la introducción a Proyectos Nacionales, 
Varsavsky señala que el libro tendrá un segundo tomo que contendrá un capítulo sobre viabilidad política, otro sobre viabilidad social y otro sobre los criterios para clasificar grupos sociales. Sin embargo, el segundo tomo no fue publicado y el autor no profundizó en el problema del cálculo de la viabilidad política en sus trabajos posteriores, aunque sí debemos señalar que la "participación popular" es una de las características centrales de su estilo de desarrollo de preferencia denominado creativo ${ }^{9}$. Uno de sus amigos y colaboradores, Alfredo Eric Calcagno, se dedicó sí especialmente a este problema, como veremos a continuación.

Hacia 1968, Alfredo Eric Calcagno, Pedro Sáinz y Juan De Barbieri publican un artículo titulado "El diagnóstico político en la planificación" en el que procuran ofrecer un modo de evaluar la viabilidad de los planes que involucre la realidad política y social, en lugar de aspectos meramente económicos. Los autores nos recuerdan que "la economía es, esencialmente, un 'arte ministerial' de la política" (Calcagno, Sainz \& De Barbieri, 1968, p. 389) y, por lo tanto, no puede planificarse la economía sin considerar cuál será la reacción de las diferentes fuerzas políticas en términos de apoyos o rechazos. Por lo demás, el diagnóstico político no puede realizarse de modo general o implícito ya que entonces se corre el riesgo de basarse en opiniones o prejuicios. Debe procurarse entonces, al igual que para Matus, una sistematización del diagnóstico político incorporándola al proceso de planificación.

Las principales reflexiones del artículo aludido fueron incorporadas en un capítulo del libro compilado por Varsavsky, América Latina: Modelos matemáticos $(1971)^{10}$. Los autores realizan advertencias similares a las realizadas por Matus en torno a la necesidad de considerar la viabilidad política: esto en modo alguno significa que lo planificado deberá responder al mantenimiento del statu quo, sino que las relaciones de poder son condicionantes del plan y deben ser tenidas en cuenta precisamente para determinar si es necesario cambiarlas.

La propuesta consiste en la construcción de un "modelo" que "reproduzca las estructuras sociales" y se considere como un "simulacro" de la realidad que permita "mostrar interrelaciones y prever consecuencias" teniendo como propósito orientar la acción (Calcagno et al., 1968, p. 390). Por otra parte, los autores reconocen la necesidad de integrar elementos económicos, sociales y políticos, pero advierten que en vistas de las dificultades operativas para construir modelos tan amplios se centrarán, por tratarse de una indagación preliminar, en los aspectos políticos del problema exclusivamente. Hasta aquí, resultan claras las similitudes con las simulaciones que Matus propone en su planificación estratégica. Sin embargo, para estos autores el método consiste precisamente en la experimentación numérica desarrollada por Oscar Varsavsky: en la traducción al lenguaje matemático de las descripciones políticas, la cuantificación de las variables y sus relaciones y el establecimiento de las operaciones que permitirán obtener los resultados del modelo. Ya nos referimos a las reservas de Matus con respecto a los modelos matemáticos, es justo indicar de todas formas que para Calcagno, Sainz y Barbieri, la formalización es la "expresión simbólica de un razonamiento" (Calcagno et al., 1968, p. 393) y, por tanto, puede contribuir a perfeccionar el esquema mental pero en modo alguno puede reemplazarlo ${ }^{11}$.

En tanto se considera al sistema político como una "constelación de fuerzas" en pugna por la realización o el rechazo a determinados actos políticos mediante su participación en el sistema por influencia o poder de persuasión, por el control de mecanismos institucionales o bien el control de facto de los mecanismos de ejecución de los actos. Las fuerzas políticas se consideran, pues, por la influencia que pueden ejercer sobre los actos, cualquiera sea su grado de institucionalización o el medio por el cual ejerzan dicha influencia. El análisis no requiere, al igual que para Matus, definir las fuerzas a priori, sino en función de su posición con respecto a los actos políticos o proyectos. Los actos de gobierno tienen valor o importancia en relación a los demás, las fuerzas políticas tienen peso o capacidad de influencia e interés en la ejecución de un acto, sea positiva o negativamente. De la contraposición de intereses resulta el antagonismo entre las fuerzas políticas. La acción refiere a la energía con que actúa una fuerza, mientras que la presión es el grado de influencia que ejerce en relación con un acto en particular. Un acto tiene a su vez un grado de solidez o firmeza que resulta de las presiones de todas las fuerzas, una cierta estabilidad o posibilidad de mantenerse y un grado de factibilidad 
que indica sus posibilidades de ser ejecutado. Entre las fuerzas existe a su vez un grado de transacción o conciliación. Por último, la compulsión refiere a la coacción que puede emplear un gobierno hasta lograr la estabilidad de un acto (Calcagno et al., 1968).

Ahora bien: el modelo debe comenzar por determinar algunos valores que quedarán establecidos a modo de supuesto para poder realizar el cálculo. Nótese que, independientemente de que se requiera o no el uso de modelos matemáticos, en la propuesta de Matus también se requiere la realización de un diagnóstico que, a partir del estudio histórico de los grupos sociales, pueda prever su posicionamiento y establecer una trayectoria. También en el caso del modelo propuesto por Calcagno y otros, se calcula en primer lugar "la factibilidad de cada uno de los actos en la secuencia en que han sido dados" (Calcagno et al., 1968, p. 400) y, en segundo lugar, las modificaciones en los pesos y los intereses de las fuerzas consecuencia de la consecución o no del acto. Nuevamente, se considera la viabilidad de una secuencia que debe evaluarse en cada una de las etapas, obligando a revisar el modelo a medida que se van realizando algunos logros (o fracasos) parciales. El modelo contempla también cuáles serán los "grados de transacción” o de negociación que se deberá realizar y con qué fuerzas (en particular, aquellas que se opongan al acto en forma débil), "pero se fija un tope a la transacción, pasado el cual el acto primitivo se habría desnaturalizado en tal grado que ya no sería el mismo" (Calcagno et al., 1968, p. 402). Lo mismo con respecto a la compulsión o la fuerza que puede usarse para imponer la consecución de un acto.

En síntesis, nos interesa señalar que, cualquiera sea la terminología utilizada, e independientemente de si se utilizan modelos matemáticos o se prefieren los esquemas cualitativos, tanto la propuesta de Calcagno y otros como la de Carlos Matus proponen un ejercicio de simulación para la toma de decisiones sobre actos o proyectos específicos, enmarcados en una estrategia o estilo de desarrollo, que tome en consideración los posicionamientos de las distintas fuerzas o grupos sociales en relación con la propuesta, sus capacidades para fortalecerla o vetarla y el modo en que una secuencia o sucesión de ejecuciones puede influir sobre el conjunto del sistema o constelaciones.

\section{Algunas Conclusiones generales}

A lo largo de este trabajo procuramos recuperar la producción de Carlos Matus de fines de la década del sesenta y principios de los setenta y ponerla en relación con los debates sobre estilos de desarrollo. Tejimos, pues, un diálogo en torno a la pregunta por la planificación del futuro y su factibilidad material y política que, como vimos, anuda con la pregunta por los estilos, modelos o estrategias de desarrollo. Entre las dimensiones que asume ese diálogo podemos señalar, en primer lugar, la búsqueda de otros futuros posibles a partir de un ejercicio de simulación que, partiendo de la realidad actual, permita definir cursos de acción viables independientemente del grado de formalización con que se realice (o bien, pese a la desconfianza de Matus hacia los modelos matemáticos). El ejercicio supone la incorporación de una gran cantidad de variables y un análisis de viabilidad para cada una de las etapas del proceso. En segundo lugar, encontramos la crítica al economicismo y la propuesta de considerar la exploración de futuro en sus aspectos económicos, sociales y políticos. Esto conlleva la impugnación de las categorías económicas tradicionales, al tiempo que supone la remisión del proceso de toma de decisiones a metas últimas sobre cuya elección no pueden realizarse objeciones técnicas, pues responden a valoraciones de carácter ideológico. La coherencia de cada una de las metas parciales también debe realizarse en función a estos objetivos últimos. Por último, lo anterior se desprende de la necesidad de pensar nuevos estilos o estrategias de desarrollo que, partiendo de un fuerte cuestionamiento al modelo propuesto por los países desarrollados, procuren la inclusión de las grandes mayorías en los procesos de producción y consumo. Esto constituye también una invitación a pensar los estilos o estrategias de desarrollo en términos que amplíen sus esferas más allá de la cuestión económica al tiempo que incorporen, en el análisis de su viabilidad, a los distintos grupos sociales que puedan fortalecer o 
bien debilitar la estrategia. Señalemos por último, a modo de conclusión, algunas líneas generales por las que podría continuar la presente indagación.

A la luz de todo lo precedente, nos vemos en una invitación a revisar las producciones posteriores de Carlos Matus. A priori, podríamos señalar que se vuelve necesario volver sobre la consideración, bastante extendida por cierto, de la planificación estratégica como una operación de autocrítica producida por Matus a su propia actuación en el gobierno de Salvador Allende. Diversos trabajos han recuperado esta lectura (Spinelli \& Testa, 2005; Bernazza, 2006; Mattos, 2010; Sotelo Maciel, 2013), incluso el propio Matus en su libro Adiós, señor presidente (1987) se hizo eco de la misma. A nuestro entender, esta interpretación puede ser puesta en tensión a la luz de las consideraciones analizadas. Quizás, no toda la planificación del desarrollo fue normativa en los términos de Matus, así como no todo "el desarrollo" fue de sesgo economicista. Una primera indagación arroja, al menos, una pluralidad de estilos, estrategias, modelos. Entendemos que profundizar en el estudio de estas cuestiones podría contribuir también a echar luz sobre posibles olvidos, silencios o desplazamientos en los desarrollos posteriores de Matus en particular y de la planificación estratégica en el ámbito público en términos generales, uno de los cuales tal vez sea precisamente el problema del desarrollo o bien la definición de proyectos nacionales en términos amplios. Cabe preguntarse si la planificación no ha sido desligada posteriormente del problema del desarrollo a partir de una vocación por ampliar sus esferas a ámbitos de la vida social que excedan lo meramente económico, en una operación que supone simultáneamente la consideración del "único" desarrollo y su planificación hasta entonces como meramente económicos. Si estas suposiciones son correctas, dicha ampliación de las esferas de incumbencia de la planificación estratégica a distintos ámbitos o esferas de gobierno con relativa independencia mutua podría significar su desligue de la remisión a una "imagen-objetivo" que abarcaba el conjunto de la vida social. En el marco de este movimiento, la participación popular también habría quedado vinculada con la circunscripción de la planificación a espacios locales o comunitarios de gobierno, en detrimento de los proyectos nacionales o regionales (Bernazza, 2006). En cualquier caso, esperamos que los “apuntes" que aquí presentamos a modo de indagaciones preliminares puedan profundizarse en estudios futuros.

\section{REFERENCIAS BIBLIOGRÁFICAS}

Aguilar, P. (2016). Planificar una 'nueva sociedad': tiempo, trabajo, política. En Grondona, A. (comp.), Estilos de desarrollo y buen vivir. Buenos Aires: Ediciones del CCC.

Bernazza, C. (2006). La planificación gubernamental en Argentina. Experiencias del periodo 1974-2000 como puntos de partida hacia un nuevo paradigma (Tesis de Doctorado en Ciencias Sociales). Buenos Aires: FLACSO.

Bernazza, C. y Longo, G. (2014). Debates sobre las capacidades estatales en Argentina: un estado del arte. Revista Estado y Politicas Públicas, 3, 107-130.

Calcagno, A. E., Sáinz, P y De Barbieri, J. (1971). Programas de gobierno y desarrollo político. En Calcagno, A. E. y Varsavsky, O. (comps.), América Latina: Modelos Matemáticos. Ensayos de aplicación de modelos de experimentación numérica a la política económica. Santiago de Chile: Editorial Universitaria.

CENDES (1971). Estilos de desarrollo. En Calcagno, A. E. y Varsavsky, O. (comps.), América Latina: Modelos Matemáticos. Ensayos de aplicación de modelos de experimentación numérica a la politica económica. Santiago de Chile: Editorial Universitaria.

CEPAL (1962). El Instituto Latinoamericano de Planificación Económica y Social. Boletín Económico de América Latina, 7(2), 121-129.

Cornblit, O., Di Tella, T. y Gallo, E. (1971). Un modelo de cambio político para América Latina. En Calcagno, A. E. y Varsavsky, O. (comps.), América Latina: Modelos Matemáticos. Ensayos de aplicación de modelos de experimentación numérica a la política económica. Santiago de Chile: Editorial Universitaria.

Coviello, R. y Pryluka, P. (2016). Las pautas de consumo como problema. En Grondona, A. (comp.), Estilos de desarrollo y buen vivir. Buenos Aires: Ediciones del CCC. 
Di Filippo, A. (2014). El ILPES: reflexiones sobre su historia y perspectivas de futuro. En Máttar, J. y Perrotti, D. E. (eds.), Planificación, prospectiva y gestión pública. Reflexiones para la agenda del desarrollo. Santiago de Chile: CEPAL.

Fiuza, P. y Viedma, C. (2016). Unidad latinoamericana y desarrollo en ALBA y Buen Vivir. Una aproximación desde la historia del presente. En Grondona, A. (comp.), Estilos de desarrollo y buen vivir. Buenos Aires: Ediciones del CCC.

García Delgado, D. y Ruiz del Ferrier, C. (2013). El nuevo paradigma. Algunas reflexiones sobre el cambio epocal. Revista Estado y Politicas Públicas, 1, 61-81.

Grondona, A. (2016). Ciencia, cientificismo y (estilos de) desarrollo. En Grondona, A. (comp.), Estilos de desarrollo y buen vivir. Buenos Aires: Ediciones del CCC.

Haidar, V. (2016). Cuestión ecológica, buen vivir y debates sobre estilos de desarrollo. En Grondona, A. (comp.), Estilos de desarrollo y buen vivir. Buenos Aires: Ediciones del CCC.

Herrera, A., Scolnick, H., Chichilnisky, G., Gallopin, G., Hardoy, J., Mosovich, D., Oteiza, E., Romero Brest, G., Suárez, C. y Talavera, L. (1977). Catástrofe o nueva sociedad: Modelo Mundial Latinoamericano. Buenos Aires: Centro Internacional de Investigaciones para el Desarrollo- América Latina.

ILPES (1967). “Actas resumidas provisionales”. Santiago de Chile, 23 y 24 de febrero de 1967.

ILPES (1969). Discusiones sobre planificación: informe de un Seminario. México: Siglo XXI.

ILPES (1970). Dos polémicas sobre el desarrollo de América Latina. Santiago de Chile: Editorial Universitaria.

Mattos, R. A. (2010). (Re)visitando alguns elementos do enfoque situacional: um exame crítico de algumas das contribuições de Carlos Matus. Ciência \& Saúde Coletiva, 15(5), 2327-2336.

Matus, C. (1965). Los sistemas de planificación y su vigencia en América Latina. En Conferencia Latinoamericana sobre la infancia y la juventud en el desarrollo nacional, ILPES, Santiago de Chile.

Matus, C. (1970a). El desarrollo del interior de América Latina: ¿tesis fantasiosa o interrogante fundamental? En ILPES, Dos polémicas sobre el desarrollo de América Latina. Santiago de Chile: Editorial Universitaria.

Matus, C. (1970b). Más sobre las tesis de 'El desarrollo del interior’: réplica a Pedro Vuskovic. En ILPES, Dospolémicas sobre el desarrollo de América Latina. Santiago de Chile: Editorial Universitaria.

Matus, C. (1970c). Reflexiones sobre una nueva estrategia latinoamericana de desarrollo. En ILPES, Dos polémicas sobre el desarrollo de América Latina. Santiago de Chile: Editorial Universitaria.

Matus, C. (1972). Estrategia y plan. México: Siglo XXI.

Neirotti, N. (2016). Políticas sociales: múltiples actores, múltiples manos. Revista Estado y Política Públicas, 6, 39-58.

Ossorio, A. (2003). Planeamiento estratégico. Buenos Aires: Subsecretaría de la Gestión Pública. Oficina Nacional de Innovación de Gestión. Dirección de Planeamiento y Reingeniería Organizacional. Instituto Nacional de la Administración Pública.

Sotelo Maciel, J. (2013). Planificación, desarrollo y capacidad política: desafíos de América Latina en el siglo XXI. Revista Estado y Politicas Públicas, 1, 47-63.

Spinelli, H. (2012). El proyecto político y las capacidades de gobierno. Salud Colectiva, 8(2), 107-130.

Spinelli, H. y Testa, M. (2005). Del Diagrama de Venn al Nudo Borromeo. Recorrido de la Planificación en América Latina. Salud Colectiva, 1(3), 323-335.

Tiranti, S. (2016). Los Dispositivos de Gestión Estatal, en busca de su sentido estratégico. Revista Estado y Politicas Públicas, 7, 123-235.

Varsavsky, O. (1971a). Proyectos nacionales. Planteo y estudios de viabilidad. Buenos Aires: Periferia.

Varsavsky, O. (1971b). Largo plazo: ¿Un solo estilo? El Trimestre Económico, 38(152).

Varsavsky, O. (1971c). Modelos matemáticos y experimentación numérica. En Calcagno, A. E. y Varsavsky, O. (comps.), América Latina: Modelos Matemáticos. Ensayos de aplicación de modelos de experimentación numérica a la política económica. Santiago de Chile: Editorial Universitaria. 
Vilas, C. M. (2012). Instituciones: ni tanto ni tan poco. Aportes para el Estado y la administración gubernamental, 30, 43-51.

\section{Notas}

1 Nos referimos, puntualmente, a la reconfiguración del rol de los Estados que se inauguró con la crisis de los gobiernos neoliberales en la región, que tuvo como correlato el cuestionamiento a las corrientes de pensamiento que hasta entonces abordaban la acción estatal. Se produce entonces un conjunto de posicionamientos que procuran acentuar el carácter estratégico de las políticas públicas, poniendo en el centro de la escena la preocupación por los determinantes políticos del accionar estatal, en detrimento de las visiones que privilegian la separación entre las definiciones políticas y el carácter técnico de su implementación (Vilas, 2012; García Delgado, 2013; Tiranti, 2016).

2 Creado en 1962 desde el seno de la Comisión Económica para América Latina (CEPAL), el ILPES tuvo financiamiento de Naciones Unidas y del Banco Interamericano de Desarrollo (BID) y sede en Santiago de Chile. La iniciativa fue promovida por Raúl Prebisch y tuvo por objeto proporcionar servicios de capacitación, asesoría e investigación (CEPAL, 1962). Su creación se produce en el marco de la legitimación de la promoción de la planificación y la fuerte intervención estatal para los países en vías de desarrollo que cristaliza en la formulación de la Alianza para el Progreso. La creación de oficinas de planificación en distintos países latinoamericanos y las exigencias para la obtención de financiamiento internacional demandaban servicios de asesoría como los que proponía el ILPES (Di Filippo, 2014).

3 Oscar Varsavsky fue un físico, químico y filósofo argentino pionero en la región en la elaboración de modelos matemáticos de experimentación numérica para el estudio de la realidad social y la exploración de alternativas. Basándose en los trabajos de Edward Holland para un modelo económico de la India, Varsavsky dirigió el primer modelo para la Argentina realizado en el Instituto del Cálculo de la Universidad de Buenos Aires. Sus trabajos circularon también en ILPES-CEPAL a través de Jorge Ahumada y Alfredo Eric Calcagno, en el Instituto Di Tella y en el Centro de Estudios para el Desarrollo (CENDES) venezolano. El modelo consistía en asignar rangos a un conjunto de variables componentes previamente definidas cuyas interconexiones se suponían a partir de modelos teóricos y sobre las cuales se proyectaban escenarios sucesivos para evaluar la factibilidad de líneas de acción diversas. Como dijimos, volveremos sobre los trabajos de Varsavsky en la segunda parte del artículo.

4 En el mismo sentido se pronunció Osvaldo Sunkel, quien también participó de la mencionada reunión: "la eficacia del proceso de planificación no se mide tanto por la perfección o imperfección de los mecanismos y de las técnicas que en él intervienen sino por la claridad y definición con que está planteada la política de desarrollo a la cual se supone que ese proceso de planificación sirve" (ILPES, 1968, p. 14). Si bien no retomaremos específicamente a este autor, resulta interesante mencionar que fue protagonista de las discusiones sobre "estilos de desarrollo" a las que nos referimos más adelante, en particular a partir de la problemática sobre el medio ambiente y la ciencia y la tecnología (Grondona, 2016 \& Haidar, 2016).

5 El primero de estos efectos o "causas aparentes" consiste en la temprana formalización institucional que se otorgó a la planificación, insertándola sobre estructuras administrativas preexistentes que se dejaron inalteradas, por lo que en ocasiones los criterios de eficacia administrativa tendieron a primar por sobre los restantes. Las reflexiones en torno a la eficacia económica, eficacia política y eficacia burocrática a las que nos referimos en el apartado anterior se producen en este marco. La pérdida de apoyo político a la planificación posterior reforzó esta característica, que se suma al "factor humano" de la falta de liderazgo de los planificadores para encauzar el proceso. Por último, la tendencia de los expertos en planificación a enfocarse en el dominio metodológico del problema, por sobre el conocimiento de los procesos económicos y político-sociales también contribuyó también a la crisis de la planificación (Matus, 1972).

6 Hemos observado que, si bien por momentos las referencias al "patrón”, "estilo" o "estrategia” de desarrollo parecían indicar que ambos términos son sinónimos, en otras secciones de los documentos la noción de "estilo" pareciera referirse a la imagen-objetivo o metas últimas, mientras que el "patrón” de desarrollo refiere al conjunto de proyectos sociales básicos seleccionados para una etapa del proceso y la "estrategia" a la puesta en secuencia de varios patrones de desarrollo sucesivos.

7 En efecto, también para Varsavsky se oponen los proyectos nacionales producidos desde los países emergentes al estilo de desarrollo "desarrollista" o "consumista" de los países centrales. Asimismo, otros participantes de los debates sobre estilos de desarrollo que no analizamos en este trabajo sí postulan la necesidad de una estrategia de desarrollo regional. Nos referimos puntualmente al Modelo Mundial Latinoamericano elaborado por Fundación Bariloche, publicado algunos años después (Herrera et al., 1977). En efecto, el problema de la integración latinoamericana adquiriría particular relevancia en estos debates (Fiuza \& Viedma, 2016).

8 Para profundizar en torno al problema del consumo en los debates sobre estilos de desarrollo puede consultarse el trabajo de Coviello y Pryluka (2016), en particular en relación al Plan Trienal para la Liberación y la Reconstrucción Nacional 
(1973), un documento que no hemos trabajado en este artículo pero en cuyas formulaciones resuenan fuertemente algunas tramas de estos debates.

9 También denominado socialismo nacional creativo y, posteriormente, estilo pueblocéntrico, en este estilo la plena participación ejercida críticamente constituía un resguardo contra los posibles riesgos de autoritarismo que involucraba una planificación tan detallada de la vida social. Al igual que para Matus, se destacaba la importancia de fomentar la "capacidad humana de creación”. Esto involucraba, a su vez, la necesidad de transformar gradual pero profundamente los modos de sociabilidad y subjetividad vigentes. Para profundizar en este último aspecto puede consultarse el trabajo de Aguilar (2016).

10 En la misma compilación, Oscar Cornblit, Torcuato Di Tella y Ezequiel Gallo publican "Un modelo de cambio político para América Latina”. Sin embargo, se trata de un modelo de simulación del proceso de cambio social y político cuyo propósito es explicar la realidad antes que analizar la viabilidad de un programa de gobierno. Por ese motivo, no lo hemos incorporado a nuestro análisis.

11 En efecto, uno de los propósitos para utilizar modelos matemáticos es precisamente la necesidad de perfeccionar los “modelos mentales" que, al requerir una gran cantidad de información, son susceptibles de presentar inconsistencias o falencias que la formalización contribuye a detectar (Varsavsky, 1971c). 